\title{
Gas-solid coexistence of adhesive spheres
}

\author{
P. Charbonneau and D. Frenkel $\left.\right|^{\mathrm{a})}$ \\ FOM Institute for Atomic and Molecular Physics, Kruislaan 407, 1098 SJ Amsterdam, The Netherlands
}

(Received 7 February 2007; accepted 12 April 2007; published online 16 May 2007)

[DOI: $10.1063 / 1.2737051]$

The Baxter limit of adhesive spheres ${ }^{1}$ is often deemed an unphysical model for liquids due to the thermodynamic metastability of its disordered phases with respect to crystal formation. $^{2,3}$ In this note we use basic free energy considerations to suggest conditions under which the disordered phases are actually stable with respect to the solid for narrow attractive interactions down to the Baxter limit. Possible realizations are then briefly discussed.

We consider the original Baxter adhesive sphere model, i.e., a three-dimensional (except where explicitly mentioned) square-well fluid with well depth $\epsilon$ and well width $\delta$, where the hard-core diameter is set to unity for convenience. The second virial coefficient is then $B_{2 v} / B_{2 v}^{\mathrm{HS}}=1-(1 / 4 \tau)$, where $B_{2 v}^{\mathrm{HS}}=2 \pi / 3$ is the hard-sphere (HS) value. The stickiness $\tau$ is a temperaturelike quantity defined as

$$
\tau^{-1} \equiv 12 \delta e^{\epsilon / k_{B} T}
$$

for adhesive spheres and as

$$
\tau^{-1}=4\left[(1+\delta)^{3}-1\right]\left(e^{\epsilon / k_{B} T}-1\right)
$$

for finite-width square wells, ${ }^{4}$ where $k_{B}$ is Boltzmann's constant and $T$ is the temperature.

The gas-liquid critical point of sticky spheres (and, to a good approximation, all fluids with a narrow, isotropic attraction $^{5}$ ) occurs at $\tau_{c} \approx 0.11$. $^{6}$ How about freezing? At low temperatures, the density of the gas coexisting with the solid is very low, $\rho_{g} \ll 1$, while at high temperatures, the freezing density is that of hard spheres. Typically, the freezing curve moves rather abruptly, but continuously, from one limit to the other. We estimate $\tau$ where this happens and compare it with the known $\tau_{c}{ }^{6}$

To obtain the crossover temperature where the vapor pressure starts to increase, we equate the chemical potential $\mu$ of the coexisting phases. For the fluid, we use an ideal gas (we check this approximation later), while we treat the crystal as a collection of uncorrelated cells each with free volume $v_{f}$. Although this last approximation is rather crude compared to what is usually used in similar systems, ${ }^{9}$ for narrow square-well potentials at low temperatures, it is not unreasonable. We also assume the volume per particle $v$ to be much smaller in the crystal than in the gas, so the difference $\Delta v \approx \rho_{g}^{-1}$. This gives

$$
\frac{\Delta \mu}{k_{B} T} \approx-\frac{Z \epsilon}{2 k_{B} T}-\ln \rho_{g} v_{f}-1,
$$

where $Z$ is the coordination number of the crystal. Note that when $\Delta \mu=0, k_{B} T_{\text {coex }} / Z \epsilon$ is the only temperature term; thus, to a first approximation changing the coordination number of the solid rescales linearly the coexistence temperature.

Following Sear, ${ }^{2}$ we take $v_{f} \approx(\delta / 2)^{d}$ for narrow square wells in $d$ dimensions, so Eq. (3) can be used to calculate the gas-solid phase coexistence directly,

$$
\frac{k_{B} T_{\mathrm{coex}}}{\epsilon}=\frac{-Z / 2}{\ln \rho_{g}(\delta / 2)^{d}+1} .
$$

This is compared with published numerical results of $Z=12$ narrow square-well phase diagrams in Fig. 1. At low $\rho_{g}$, there is fairly good agreement between this crude treatment and the Monte Carlo simulations. Results for $Z=4$ also qualitatively agree with this picture. ${ }^{10}$

As a last consistency check, we examine in what regime corrections to $\Delta v \approx \rho_{g}^{-1}$ are small. The next order terms are the crystal's finite volume, which is of $\mathcal{O}(1)$, and the fluid's second virial coefficient $B_{2 v}$. Using Eq. (4) as a mean-fieldlike closure for $\epsilon / T_{\text {coex }}$ gives

$$
\frac{B_{2 v}^{\text {coex }}}{B_{2}^{\mathrm{HS}}} \approx 1-3 \delta\left(e^{\epsilon / T_{\mathrm{coex}}}-1\right) \approx 1-3 \delta\left[\left(\frac{8}{e \delta^{3} \rho_{g}}\right)^{2 / Z}-1\right],
$$

which for $Z \geqslant 6$ leaves an $\mathcal{O}\left(\rho_{g}^{-2 / Z}\right)$ correction. At low densities, the contributing factors are both much less than $\rho_{g}^{-1}$ and have opposite signs, so they amount to an error in $T_{\text {coex }}$ of less than a few percent for narrow well widths. This level of precision is sufficient for the sake of our argument. It also stays clear of the dense-fluid regime, where the cell treatment

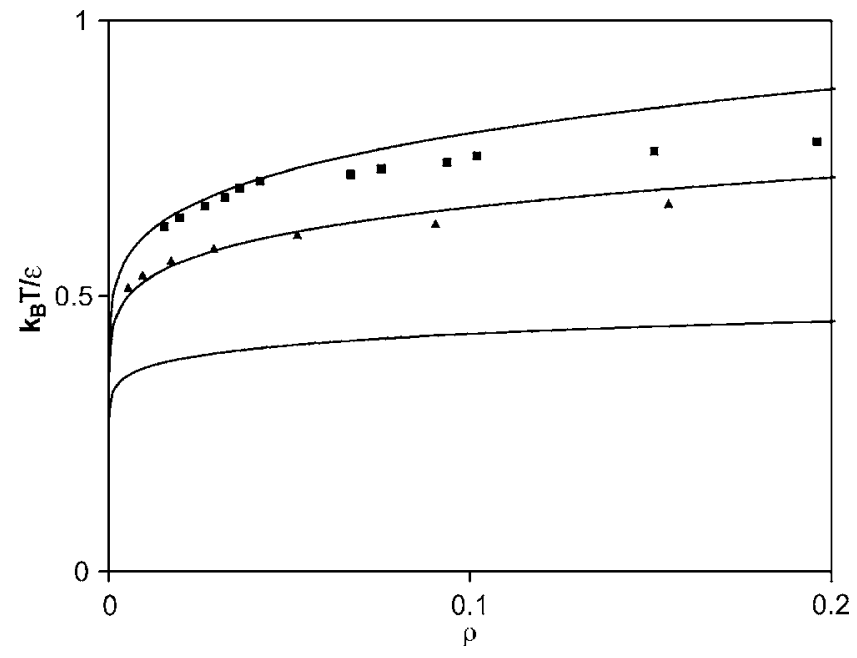

FIG. 1. Gas-solid coexistence line from Eq. (4) for square-well interactions with $Z=12$ and $\delta=0.25,0.15$, and 0.03 , from top to bottom. Simulation results for the first two cases are included for comparison (Refs. 7 and 8). 


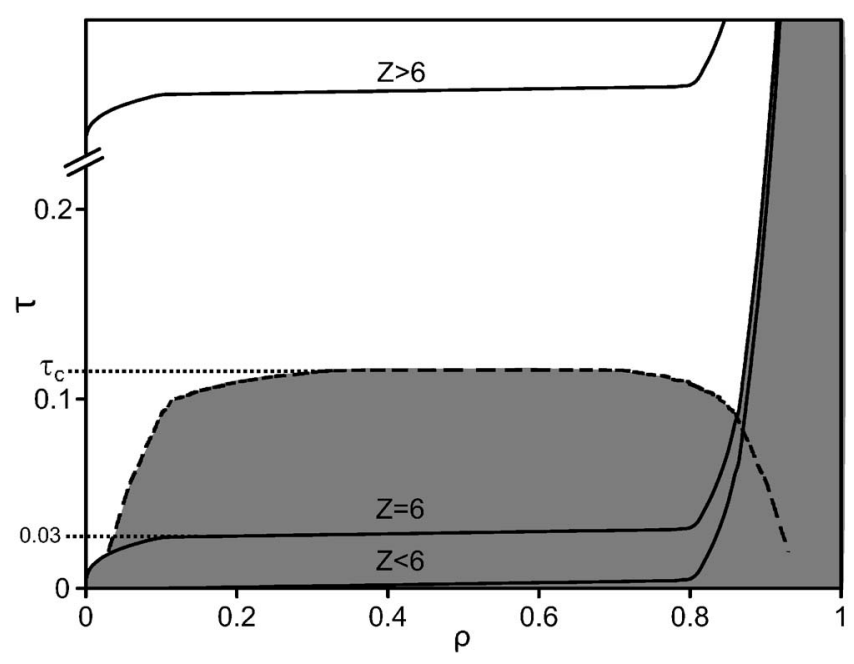

FIG. 2. Sketch of the three-dimensional Baxter phase diagram following Ref. 6 for the isotropic gas-liquid binodal (dashed line) and this work for the gas-solid coexistence curves (solid lines). The gas-solid coexistence is found at high $\tau$ for $Z>6$ and at very low $\tau$ for $Z<6$, while it follows Eq. (7) for $Z=6$. Coexistence curves of gas with solid for $\rho \gtrsim 0.1$ and gas with liquid for $\tau<0.1$ are drawn for schematic purposes only.

is known to break down and lead to a nonphysical liquidliquid critical point. ${ }^{11}$

In the Baxter $\delta \rightarrow 0$ limit, we use the stickness from Eq. (1) to rewrite Eq. (4) as

$$
1=-\ln \left[2^{-d} \rho_{g} \delta^{d-Z / 2}\left(\frac{1}{12 \tau_{\text {coex }}}\right)^{Z / 2}\right] .
$$

For $Z>2 d, \delta^{d-Z / 2}$ diverges, while at freezing the right-hand side of Eq. (6) should be $\mathcal{O}(1)$. If we fix the density of the gas at a small but finite value, it follows that $\tau_{\text {coex }} \rightarrow \infty$-i.e., the fluid freezes well above $\tau_{c} \approx 0.11$. Conversely, when $Z$ $<2 d, \tau_{\text {coex }}=0$, so the triple point also lies on the $\tau=0$ axis. The interesting case is the marginal situation $Z=2 d$, where we obtain a nontrivial fixed crossover $\tau_{\text {coex }}$. Indeed, for $d$ $=3$, the crossover $\tau_{\text {coex }}$ increases by less than $15 \%$ as $\delta$ varies from 0.25 down to the Baxter limit, which is small relative to the divergence obtained for $Z>2 d$. Also, in this case Eq. (6) reduces to

$$
\tau_{\text {coex }}=\frac{\left(e \rho_{g}\right)^{1 / 3}}{24} .
$$

Using $\rho_{g}=0.1$ as a reference point, we obtain a crossover $\tau_{\text {coex }} \approx 0.03$, which is well below $\tau_{c}$ for the isotropic case, as shown in Fig. 2. The gas-liquid critical point is thus stable with respect to the crystal.
Simple cubic crystals, among others, as well as isostatic solids fulfill the $Z=2 d$ condition for all $d$. Though the analysis presented above is not specific to solid crystalline forms, it does require the solid phase to be mechanically stable for the cell treatment to be valid. Besides, if the solid considered is not the thermodynamic ground state, its metastability ought to be sufficiently long-lived to allow for the vapor pressure to equilibrate. Monodisperse, isotropically adhesive spheres invariably crystallize in $Z=12$ phases, but if either the spheres are polydisperse or if the interaction is patchy, a lower $Z$ can be obtained. In the adhesive limit, even infinitesimally polydisperse solids thermodynamically fractionate, ${ }^{2}$ which results in $Z \approx 12$. However, this is kinetically nearly impossible and therefore of little experimental relevance. Instead, an isostatic polydisperse metastable crystal would leave $\tau_{c}$ stable with respect to the gas-solid coexistence. For patchy adhesive spheres, $Z$ is precisely controlled. Though $\tau_{c}$ also varies with $Z{ }^{12}$ the reduced surface coverage is unlikely to be sufficient to bring it below $\tau_{\text {coex }}$.

In conclusion, if one manages to reduce the bonding of the solid phase down to $Z \leqslant 2 d$, the high density fluid remains stable with respect to the solid down to the Baxter limit. Based on rough but reasonable estimates, the threedimensional gas-liquid critical point would then also be stable with respect to crystallization. Furthermore, this scheme might be realizable both experimentally and through simulation.

One of the authors (P.C.) thanks D. R. Reichman for insightful discussions and acknowledges EU support (Contract No. MIF1-CT-2006-040871). This work is part of the research program of the "Stichting voor Fundamenteel Onderzoek der Materie (FOM)," which is financially supported by the "Nederlandse organisatie voor Wetenschappelijk Onderzoek (NWO)."

\footnotetext{
${ }^{a)}$ Electronic mail: frenkel@amolf.nl

${ }^{1}$ R. J. Baxter, J. Chem. Phys. 49, 2770 (1968).

${ }^{2}$ R. P. Sear, Mol. Phys. 96, 1013 (1999).

${ }^{3}$ G. Stell, J. Stat. Phys. 63, 1203 (1991).

${ }^{4}$ M. A. Miller and D. Frenkel, J. Phys.: Condens. Matter 16, S4901 (2004).

${ }^{5}$ M. G. Noro and D. Frenkel, J. Chem. Phys. 113, 2941 (2000).

${ }^{6}$ M. A. Miller and D. Frenkel, Phys. Rev. Lett. 90, 135702 (2003).

${ }^{7}$ D. L. Pagan and J. D. Gunton, J. Chem. Phys. 122, 184515 (2005).

${ }^{8}$ H. Liu, S. Garde, and S. Kumar, J. Chem. Phys. 123, 174505 (2005).

${ }^{9}$ C. F. Tejero, A. Daanoun, H. N. W. Lekkerkerker, and M. Baus, Phys. Rev. E 51, 558 (1995).

${ }^{10}$ Z. Zhang, A. S. Keys, T. Chen, and S. C. Glotzer, Langmuir 21, 11547 (2005).

${ }^{11}$ J. Magee and N. Wilding, Mol. Phys. 100, 1641 (2002).

${ }^{12}$ E. Bianchi, J. Largo, P. Tartaglia, E. Zaccarelli, and F. Sciortino, Phys. Rev. Lett. 97, 168301 (2006).
} 